\title{
ENDOSCOPIC BILIARY DRAINAGE FOR SEVERE ACUTE CHOLANGITIS
}

\author{
Edward C.S. Lai, F.R.C.S.(Ed.), F.R.A.C.S., Francis P.T. Mok, F.R.C.S.(Ed.), F.R.A.G.S., \\ Eliza S.Y. Tan, R.N., Ghung-Mau Lo, F.R.C.S.(Ed.), Sheung-tat Fan, F.R.C.S.(Glas.), \\ Kok-tJang You, M.D., and John Wong, Ph.D., F.R.A.C.S., F.R.C.S.(Ed.)
}

\begin{abstract}
Background. Emergency surgery for patients with severe acute cholangitis due to choledocholithiasis is associated with substantial morbidity and mortality. Because recent results suggested that emergency endoscopic drainage could improve the outcome of such patients, we undertook a prospective study to determine the role of this procedure as initial treatment.

Methods. During a 43-month period, 82 patients with severe acute cholangitis due to choledocholithiasis were randomly assigned to undergo surgical decompression of the biliary tract (41 patients) or endoscopic biliary drainage (41 patients), followed by definitive treatment. Hospital mortality was analyzed with respect to the use of endoscopic biliary drainage and other clinical and laboratory findings. Prognostic determinants were studied by linear discriminant analysis.

Results. Complications related to biliary tract decompression and subsequent definitive treatment developed
\end{abstract}

$\mathrm{T}$ HE use of endoscopic papillotomy and nasobiliary drainage, either alone or in combination, is a satisfactory alternative to emergency exploration of the common bile duct in the treatment of patients with acute cholangitis due to choledocholithiasis. ${ }^{1-6}$ Although surgery has been the conventional treatment for these patients, remarkably low mortality rates of 4.7 and 7.6 percent have been reported in patients treated by endoscopy. ${ }^{1,4}$ Since these encouraging results of endoscopy were obtained in retrospective or prospective but uncontrolled studies, the advantage of endoscopic drainage remains unproved. Indeed, in a selected group of patients who had emergency surgery, we found a mortality rate of 5.5 percent, comparable to the rates in patients treated by interventional endoscopy ${ }^{6,7}$ We therefore conducted a randomized, controlled trial to define the benefits of emergency endoscopic drainage in patients with acute cholangitis due to choledocholithiasis.

\section{Methods}

\section{Selection of Patients}

Between November 1987 and June 1991, we studied 82 of the 96 patients with severe acute cholangitis due to choledocholithiasis seen at our institution. Forty were men, and 42 were women. Their mean age was 69 years (range, 35 to 97). Of the 14 patients not studied, 2 declined to participate, 1 was pregnant, 4 were moribund, 6 had intrahepatic stones that were not amenable to endoscopic drainage, and 1 had previously undergone Polya's gastrectomy, which made biliary drainage impossible. These patients were part of a larger group of 494 patients who had acute cholangitis, defined as the presence of fever (temperature, $>37.5^{\circ} \mathrm{C}$ ) and a history of chills, with abdominal pain, jaundice, or both. The diagnosis of severe acute cholangitis was based on the presence of either septicemic shock or evidence of progressive biliary sepsis including mental confusion and persistent or relapsing fever despite appropriate antibiotic treatment. The decision to undertake urgent biliary decom- in 14 patients treated with endoscopic biliary drainage and 27 treated with surgery ( 34 vs. 66 percent, $P>0.05$ ). The time required for normalization of temperature and stabilization of blood pressure was similar in the two groups, but more patients in the surgery group required ventilatory support. The hospital mortality rate was significantly lower for the patients who underwent endoscopy (4 deaths) than for those treated surgically ( 13 deaths) ( $10 \mathrm{vs.} 32$ percent, $\mathrm{P}<0.03)$. The presence of concomitant medical problems, a low platelet count, a high serum urea nitrogen concentration, and a low serum albumin concentration before biliary decompression were the other independent determinants of mortality in both groups.

Conclusions. Endoscopic biliary drainage is a safe and effective measure for the initial control of severe acute cholangitis due to choledocholithiasis and to reduce the mortality associated with the condition. (N Engl J Med 1992;326:1582-6.)

pression was made by two independent clinicians as well as the principal investigator. Emergency endoscopic retrograde cholangiopancreatography was performed in all patients to delineate the biliary tract before randomization while preparations for emergency surgical exploration were being made.

The protocol was approved by the ethics committee of the Faculty of Medicine of the University of Hong Kong, and informed consent was obtained from each patient or (for patients who were mentally confused) the next of kin.

\section{Randomization}

Eligible patients were randomly assigned to either surgery or endoscopic biliary drainage by drawing consecutively numbered envelopes that contained the assigned treatment for initial biliary decompression followed by appropriate elective definitive treatment.

\section{Treatment Procedure}

Emergency endoscopy was conducted with the patient under local pharyngeal anesthesia, supplemented with intravenous fatemulsified diazepam when necessary. The blood pressure, pulse rate and rhythm, and oxygen saturation were closely monitored throughout the procedure. Oxygen was routinely given through nasal prongs. Hyoscine $N$-butylbromide ( $N$-butylscopolammonium bromide) was given intravenously to relax the duodenum. Through a side-view duodenoscope (model JF-1T10 or JF-1T20, Olympus), the common bile duct was cannulated directly with an endoscopic papillotome (model $\mathrm{KD} 18 \mathrm{Q}$ or $\mathrm{KD} 20 \mathrm{Q}$, Olympus). The biliary ductal system was opacified by the gentle injection of dilute contrast medium mixed with gentamicin ( $1 \mathrm{mg}$ per milliliter). After the underlying ductal pathologic features had been delineated, an envelope was drawn for randomization. The endoscopic procedure was concluded at this stage if the patient was assigned to emergency surgery.

If the patient was assigned to receive endoscopic drainage, a small (less than $0.5 \mathrm{~cm}$ ) papillotomy was made to allow the introduction of a 7-French nasobiliary catheter (ENBD7-Liguory, Wilson-Cook Medical). The endoscope was considered to have been properly positioned for drainage when the tip of the catheter was placed proximal to the site of the obstruction (Fig. 1). No attempt was made to extract the stone or stones at this time. After the cholangitis subsided, the patient was treated by either surgery or completion of the endoscopic papillotomy, depending on general condition and whether the patient had had a cholecystectomy in the past.

All the patients assigned to surgery underwent emergency explo- 


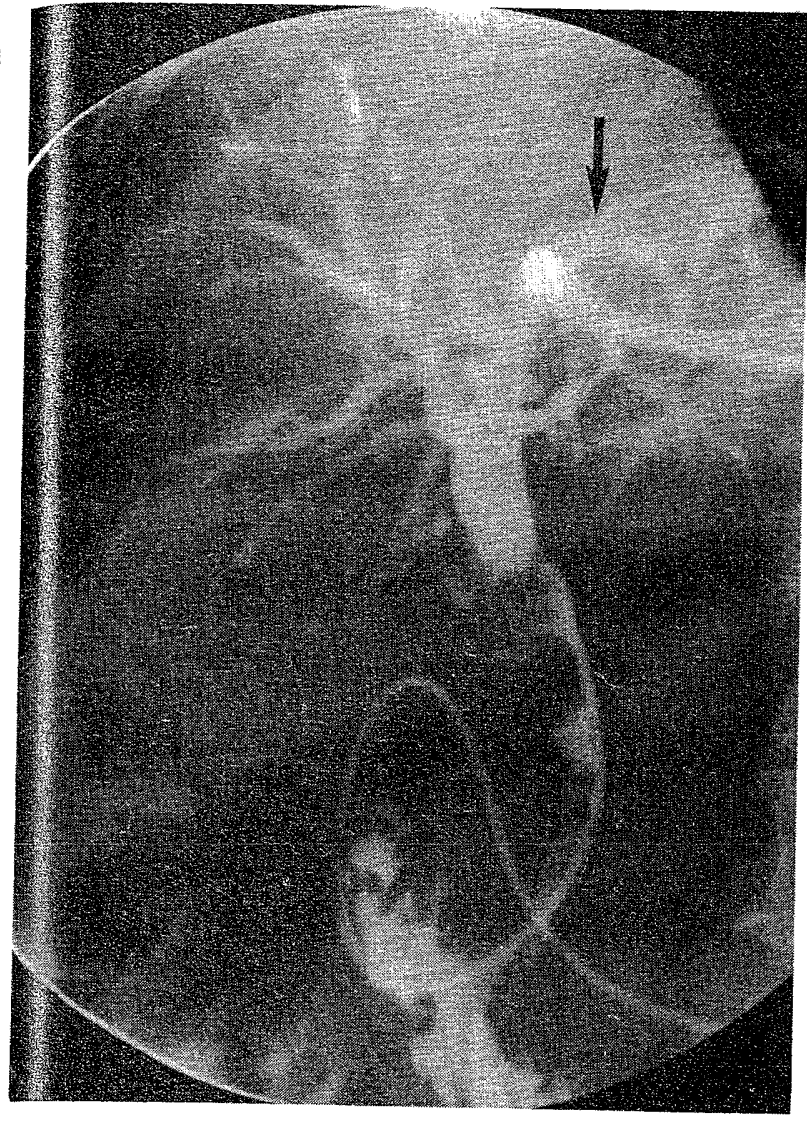

Figure 1. Cholangiogram Obtained after Successful Endoscopic Biliary Drainage in a Patient with Severe Acute Cholangitis.

Note the tip of the catheter in the left intrahepatic duct (arrow) proximal to the stones in the distal common duct.

ration of the common bile duct as soon as possible after the conclusion of the diagnostic cholangiopancreatography. The extent of ductal exploration depended on the patient's hemodynamic status. Neither in traoperative choledochoscopy nor postexploratory T-tube cholangiography was routinely performed, since the patients were often critically ill.

\section{Clinical and Laboratory Determinations}

The following clinical and laboratory values were collected: maximal temperature; duration of fever (defined as a temperature $\geqslant 37.5^{\circ} \mathrm{C}$ ); lowest systolic blood pressure recorded before emergency. biliary drainage and, depending on the treatment, at the time of induction of anesthesia or endoscopic biliary decompression; duration of hypotension (defined as systolic blood pressure $\leqslant 100$ $\mathrm{mm} \mathrm{Hg}$ ) before ductal drainage and the results of treatment for hypotension; time between admission and randomization; hemoglobin level and leukocyte and platelet counts (complete blood count); serum bilirubin, alkaline phosphatase, alanine aminotransferase, and aspartate aminotransferase levels (liver-function tests); serum urea and creatinine levels at presentation and within 24 hours before exploration; and arterial blood gas values at randomization. The patients were also evaluated for a history of biliary tract surgery, and cultures of blood and bile were obtained. Concomitant medical problems, which included ischemic heart disease, chronic obstructive airway disease, diabetes mellitus, and cancer, were assumed to be of equal weight for analysis. The location of biliary calculi and benign strictures, the diameter and tension of the common bile duct at the time of drainage, and the appearance of aspirated bile were also recorded.

We calculated each patient's risk score using the discriminant equation developed as a result of our study of 86 consecutive pa- tients who had emergency surgery for severe acute cholangitis. ${ }^{7}$ The operative risk of the patients was considered to be high if three or more of the following five factors were present and low if two or fewer factors were present: a concomitant medical problem, arterial blood $\mathrm{pH}$ of less than 7.40 , serum bilirubin concentration of more than $90 \mu \mathrm{mol}$ per liter, platelet count of less than $150 \times 10^{9}$ per liter, and serum albumin concentration of less than $30 \mathrm{~g}$ per liter. ${ }^{7}$

Hospital mortality was defined as death during the hospitalization for severe acute cholangitis. If the patient died within 48 hours after emergency ductal drainage, death was attributed to the cholangitis in the absence of another obvious contributory cause. Renal failure was diagnosed if the serum creatinine concentration doubled or exceeded $180 \mu \mathrm{mol}$ per liter among patients with normal preoperative values ( $\leqslant 130 \mu \mathrm{mol}$ per liter) or if it increased by $10 \mu \mathrm{mol}$ per liter above an abnormal preoperative value. ${ }^{8}$ The analysis of overall morbidity included any complications that developed after emergency biliary decompression and subsequent definitive therapy. Because of the urgency of the biliary drainage, residual stones were not considered a complication. Resolution of the episode of cholangitis after ductal drainage was defined as the normalization of body temperature (to $\leqslant 37.5^{\circ} \mathrm{C}$ ) or stabilization of blood pressure (systolic blood pressure, $>100 \mathrm{~mm} \mathrm{Hg}$ ) for at least eight hours. The length of time required for resolution was measured from the time of biliary decompression.

\section{Statistical Analysis}

The necessary sample size was estimated on the assumption that the hospital mortality rates after emergency surgery and endoscopic biliary drainage were 40 percent ${ }^{1,7,9-11}$ and 10 percent, ${ }^{2,6}$ respectively. Thirty-eight patients were needed in each group to have a Type I error of less than 5 percent and a Type II error of less than 20 percent with a two-tailed test. ${ }^{12}$

The statistical tests used included Student's t-test, the MannWhitney U test, the chi-square test with Yates' correction, and Fisher's exact test. All prognostic determinants of hospital mortality that were identified by univariate analysis were subjected to linear discriminant analysis to validate the influence of emergency endoscopic drainage. All the patients were analyzed according to the treatment to which they were randomly assigned (intention-totreat principle) unless otherwise stated. All the statistical calculations were conducted with standardized biomedical statistical programs (SPSS/PC+, SPSS, Chicago)

\section{Results}

\section{Clinical Features}

Seventy-seven of the 82 patients had jaundice (serum total bilirubin level, $\geqslant 26 \mu \mathrm{mol}$ per liter), 55 had a maximal body temperature of $38.3^{\circ} \mathrm{C}$ or higher, and 65 had abdominal pain. Forty-three patients had all three of these manifestations of cholangitis. Seventeen patients had mental confusion: 13 of those treated with endoscopic biliary decompression and 4 of those treated surgically $(\mathrm{P}<0.03)$. Forty-nine patients $(23$ in the endoscopic-drainage group and 26 in the surgery group) had septicemic shock, and 37 of them had hypotension within the first 24 hours after admission. At the time of the drainage procedure, 31 of these 49 patients (14 in the surgery group and 17 in the endoscopic-drainage group) continued to have hypotension despite resuscitation. Shock developed in two additional patients in the surgery group while they were awaiting operation.

Nineteen patients in each treatment group had concurrent medical problems. Twenty-five had had a biliary tract operation: 18 had had cholecystectomy and 7 cholecystectomy and exploration of the common bile duct. Fifty-two of the 82 patients entered the study within 24 hours after admission to the hospital. Four 
Table 1. Clinical and Laboratory Characteristics of 82 Patients with Severe Acute Cholangitis at the Time of Assignment to Treatment with Surgery or Endoscopic Biliary Drainage.*

\begin{tabular}{|c|c|c|}
\hline ChaRACTERISTIC & $\begin{array}{l}\text { SURGERY GROUP } \\
\quad(N=41)\end{array}$ & $\begin{array}{l}\text { ENDOSCOPIC-DRAINAGE } \\
\text { Group }(\mathrm{N}=41)\end{array}$ \\
\hline Age (yr) & $69(65-73)$ & $69(64-74)$ \\
\hline $\operatorname{Sex}(M / F)$ & $20 / 21$ & $20 / 21$ \\
\hline History of temperature $\geqslant 38.3^{\circ} \mathrm{C}$ (no. of patients) & 31 & $=24$ \\
\hline History of hypotension (no. of patients) $\dagger$ & 26 & 23 \\
\hline Lowest blood pressure $(\mathrm{mm} \mathrm{Hg}$ ) & $96(88-104)$ & $102(94-109)$ \\
\hline $\begin{array}{l}\text { Blood pressure at surgery or endoscopic } \\
\text { drainage (mm } \mathrm{Hg}) \frac{\dot{t}}{\leftarrow}\end{array}$ & $126(115-138)$ & $113(105-120)$ \\
\hline $\begin{array}{l}\text { Duration of hypotension before surgery } \\
\text { or endoscopic drainage (hr) }\end{array}$ & $2(1-3)$ & $2(1-2)$ \\
\hline Time from admission to randomization $(\mathrm{hr})$ & $27(20-37)$ & $23(15-30)$ \\
\hline Hemoglobin (g/liter) & $120(120-130)$ & $130(120-130)$ \\
\hline Hematocrit & $0.36(0.34-0.38)$ & $0.37(0.35-0.39)$ \\
\hline Leukocyte count $\left(10^{-9} /\right.$ liter $)$ & $19(15-23)$ & $18(14-21)$ \\
\hline Platelet count $\left(10^{-9} /\right.$ liter $)$ & $139(109-168)$ & $153(125-180)$ \\
\hline Serum urea nitrogen (mmol/liter) & $10(8-11)$ & $9(7-11)$ \\
\hline Serum creatinine ( $\mu \mathrm{mol} / \mathrm{liter})$ & $140(120-160)$ & $130(100-160)$ \\
\hline Serum total bilirubin $(\mu \mathrm{mol} / \mathrm{liter})$ & $137(91-183)$ & $130(109-151)$ \\
\hline Serum alkaline phosphatase (IU/liter) & $258(201-316)$ & $333(27-406)$ \\
\hline Serum alanine aminotransferase (IU/liter) & $155(110-200)$ & $202(146-259)$ \\
\hline Serum aspartate aminotransferase (IU/liter) & $184(138-230)$ & $223(169-277)$ \\
\hline Serum albumin (g/liter) & $34(32-36)$ & $35(33-37)$ \\
\hline Arterial pH & $7.44(7.42-7.46)$ & $7.42(7.40-7.45)$ \\
\hline Risk score§ & $-0.47(-0.82$ to +0.13$)$ & $-0.52(-0.91$ to +0.14$)$ \\
\hline No. of patients at high risk & 15 & 13 \\
\hline
\end{tabular}

*Values are means, with 95 percent confidence intervals in parentheses. The normal ranges of the laboratory values are as follows: hemoglobin, 118 to $170 \mathrm{~g}$ per liter; hematocrit, 0.35 to 0.51 ; leukocyte count, $3.5 \times 10^{9}$ to $9.8 \times 10^{9}$ per liter; platelet count, $140 \times 10^{9}$ to $355 \times 10^{9}$ per liter; serum urea nitrogen, 3.1 to 7.4 mmol per liter; serum creatinine, 82 to $126 \mu$ mol per liter; serum total bilinubin, 7 to $19 \mu \mathrm{mol}$ per liter; serum alkaline phosphatase, 49 to $138 \mathrm{IU}$ per liter; serum alanine aminotransferase, 13 to $33 \mathrm{IU}$ per liter; serum aspartate aminotransferase, 6 to $53 \mathrm{IU}$ per liter; serum albumin, 44 to $56 \mathrm{~g}$ per liter; and arterial $\mathrm{pH}, 7.35$ to 7.45 .

†Hypotension was defined as systolic blood pressire of $\leqslant 100 \mathrm{~mm} \mathrm{Hg}$.

\$The risk score was calculated according to the following formula: 0.27 (platelet count) $\times 10^{-2}+0.51$ (albumin level) $\times$ $10^{-1}-1.3$ (concomitant medical problems) $-0.72\left(\log _{10}\right.$ bilirubin level $)+9.2(\mathrm{pH})-69$.

of the remaining 30 patients entered the study 72 or more hours after admission. The clinical and laboratory findings in the 82 patients at the time of randomization are shown in Table 1.

\section{Operative Findings and Procedures}

All 82 patients had common-duct calculi. In addition, eight patients had stones in the hepatic ducts. Eight patients, five treated surgically and three

Table 2. Clinical Coursè after Surgery or Endoscopic Biliary Drainage in 82 Patients with Severe Acute Cholangitis.*

\begin{tabular}{|c|c|c|}
\hline VARIABLE & $\begin{array}{l}\text { SURGERY GROUP } \\
(\mathrm{N}=41)\end{array}$ & $\begin{array}{c}\text { ENDOSCOPIC-DrainaGe } \\
\text { GRouP }(N=41)\end{array}$ \\
\hline & \multicolumn{2}{|r|}{ hours } \\
\hline $\begin{array}{l}\text { Time to normalization } \\
\text { of temperature }\end{array}$ & $38(22-54)$ & $26(15-36)$ \\
\hline $\begin{array}{l}\text { Time to stabilization } \\
\text { of blood pressure }\end{array}$ & $10(5-15)$ & $10(6-13)$ \\
\hline $\begin{array}{l}\text { Duration of ventilatory } \\
\text { support }\end{array}$ & $80(27-134)$ & $47(19-75) \dagger$ \\
\hline Duration of fasting & $80(55-106)$ & $51(40-63) \dagger$ \\
\hline
\end{tabular}

*Values are means, with 95 percent confidence intervals in parentheses. $+\mathrm{P}<0.05$, for the comparison with the surgery group. $\ddagger \mathrm{P}<0.05$.

who underwent endoscopic biliary drainage, had strictures of the biliary tract. These strictures were in the common bile duct (two patients), the common hepatic duct (one), the origin of the right hepatic duct (two), and the origin of the left hepatic duct (three). Two patients in each group had empyema of the gallbladder. On the basis of the findings at the time of intervention, the bile was grossly turbid in 33 patients (13 in the endoscopicdrainage group and 20 in the surgery group) and purulent in 24 patients (13 in the endoscopicdrainage group and 11 in the surgery group).

All 41 patients in the endoscopic-drainage group had successful ductal drainage with no complications. In four patients, papillotomy was not needed for insertion of the nasobiliary catheter. Twenty-five patients had further elective endoscopic intervention as definitive therapy. Twelve patients later underwent elective cholecystectomy and exploration of the common duct, and four patients had bilioenteric reconstruction for stricture. In the surgery group, all 41 patients underwent exploration of the common duct under general anesthesia a mean of two hours after randomization. Additional procedures in this group included cholecystectomy (24 patients), cholecystostomy (2), and transhepatic intubation (1).

\section{Microbiologic Findings}

Bile and blood were collected for culture during the emergency drainage procedure in 79 of the 82 patients. Sixty-three patients ( 80 percent) had bacteria isolated from their bile, and 50 patients (63 percent) had a positive blood culture. A mixed growth of bacteria was the most frequent finding from both sources. Twenty patients had the same organisms isolated from the two sites. In nine patients (five in the surgery group and four in the endoscopic-drainage group), both bile and blood cultures were negative.

\section{Outcome, Morbidity, and Mortality}

After emergency biliary decompression, more patients in the surgery group than in the endoscopicdrainage group needed ventilatory support ( 26 vs. 12 patients, $\mathbf{P}<0.005)$. Furthermore, the patients in the surgery group needed ventilatory support for a longer time (Table 2). The time required for normalization of temperature and stabilization of blood pressure was comparable in the two groups. 
Table 3. Complications after Surgery or Endoscopic Biliary Drainage in 82 Patients with Severe Acute Cholangitis.

\begin{tabular}{|c|c|c|}
\hline COMPLICATION & $\begin{array}{c}\text { SURGERY } \\
\text { GROUP } \\
(\mathrm{N}=41)\end{array}$ & $\begin{array}{c}\text { ENDoscopic- } \\
\text { DraINAGE Group } \\
(\mathrm{N}=41)\end{array}$ \\
\hline & \multicolumn{2}{|c|}{ no. of patients } \\
\hline Heart failure & 1 & 3 \\
\hline Bronchopneumonia & 15 & 7 \\
\hline Wound dehiscence & 1 & 0 \\
\hline Wound infection & 7 & 1 \\
\hline Gastrointestinal bleeding & 2 & 0 \\
\hline Renal dysfunction & 11 & 7 \\
\hline Bleeding after papillotomy & 0 & 1 \\
\hline $\begin{array}{l}\text { Disseminated intravascular } \\
\text { coagulopathy }\end{array}$ & 2 & 0 \\
\hline Total* & 27 & 14 \\
\hline
\end{tabular}

ary drainage, but his general condition remained poor and he died of bronchopneumonia one week after exploration of the common duct.

\section{Analysis of Hospital Mortality}

In addition to the use of endoscopic biliary drainage as initial treatment, 10 other clinical and laboratory factors significantly affected the outcome of these 82 patients (Table 5). When subjected to multivariate analysis, five factors, including the use of therapeutic endoscopy, were identified as independent predictive factors, with the discriminant equation as follows:

$$
\begin{aligned}
\mathrm{R}= & 0.8 \text { (mode of drainage) }-1.2 \text { (concomitant medical problems) } \\
& +0.33 \text { (platelet count }) \times 10^{-2}-0.75(\text { serum urea level }) \times 10^{-1} \\
& +0.76 \text { (serum albumin level }) \times 10^{-1}-2.27,
\end{aligned}
$$

where $R$ is the function producing the best separation between the patients who died (mean value, 1.57) and
Fourteen patients (34 percent) in the endoscopic-drainage group and 27 (66 percent) in the surgery group had complications after biliary decompression $(\mathrm{P}>0.05)$ ( $\mathrm{Ta}$ ble 3 ). Residual stones were significantly less frequent in the patients who underwent endoscopic biliary drainage than in the patients treated surgically ( 3 vs. $12, \mathrm{P}<0.03$ ). There were 17 deaths (21 percent): 4 in the endoscopic-drainage group and 13 in the surgery group $(\mathbf{P}<0.03)$ (Table 4). Of the four patients who died in the endoscopic-drainage group, three died after subsequent definitive endoscopic intervention. The fourth patient, who had stones in both the common bile duct and right intrahepatic duct, was operated on five days after successful resolution of his biliary sepsis by endoscopic bili-
Table 5. Factors Affecting Hospital Mortality after Surgery or Endoscopic Biliary Drainage in 82 Patients with Severe Acute Cholangitis.

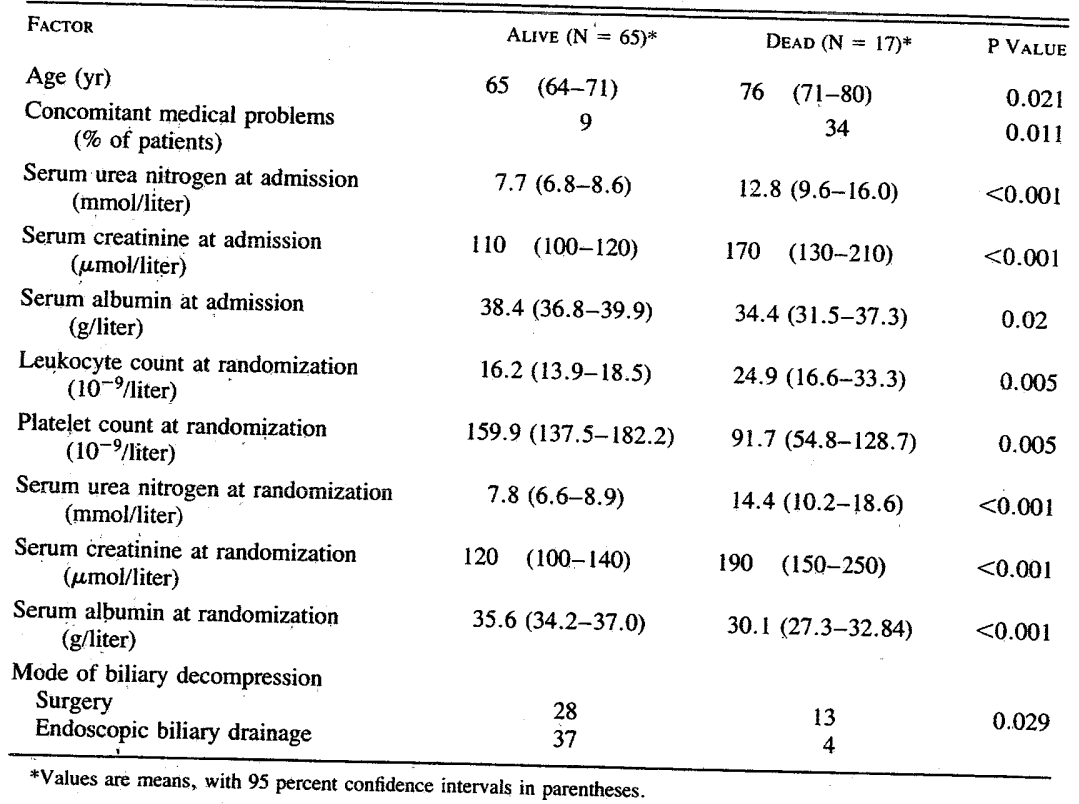

*Values are means, with 95 percent confidence intervals in parentheses.
Table 4. Causes of Death in Patients with Severe Acute Cholangitis Treated with Surgery or Endoscopic Biliary Drainage.

\begin{tabular}{lcc}
\hline CAUSE & $\begin{array}{c}\text { SURGERY } \\
\text { GRour } \\
(\mathrm{N}=41)\end{array}$ & $\begin{array}{c}\text { ENDOSCOPIC- } \\
\text { DRAINAGE GRouP } \\
(\mathrm{N}=41)\end{array}$ \\
Heart failure & & no. of patients \\
Bronchopneumonia & 2 & 0 \\
Renal failure & 2 & 2 \\
Multiorgan failure & 1 & 0 \\
Sepsis & 4 & 2 \\
Cerebellar hemorrhage & 3 & 0 \\
Total & 1 & 0 \\
& 13 & 4 \\
\hline
\end{tabular}

those who survived (mean value, 0.36). The value for mode of drainage was 0 for emergency surgery and 1 for endoscopic biliary drainage, and the laboratory results used in the analysis were those at the time of randomization. The sensitivity was 76 percent, the specificity 91 percent, and the percentage of correctly classified cases 88 percent.

When the platelet count was considered, a significant cutoff point was identified at a value of less than $110 \times 10^{9}$ per liter (relative risk, 3.6). The presence of thrombocytopenia correlated significantly with the lowest systolic blood pressure recorded before drainage (mean, $80 \mathrm{~mm} \mathrm{Hg}$ among those who died [95 percent confidence interval, 80 to 99] vs. $103 \mathrm{~mm} \mathrm{Hg}$ among those who survived [95 percent confidence interval, 97 to 110$] ; \mathrm{P}<0.02$ ) and the duration of hypo- 
tension (mean, 3 hours among those who died [95 percent confidence interval, 2 to 4] vs. 1 hour among those who survived [95 percent confidence interval, 1 to 2 ]; $\mathrm{P}<0.01$ ).

\section{Discussion}

The mortality rate among patients with severe acute cholangitis treated surgically ranges from 20 to 60 percent. ${ }^{1,7,9-11,13}$ The value of routine nonoperative biliary drainage in patients with fulminating calculous cholangitis remains controversial. In one study, none of 16 patients died after initial percutaneous biliary decompression,${ }^{14}$ whereas in another study the mortality rate was comparable to that in patients who had a staged operation and in those who underwent emergency surgery. ${ }^{15}$ The value of endoscopy in reducing the mortality associated with severe acute cholangitis is demonstrated by this prospective, randomized, controlled trial. In addition to a significantly reduced mortality rate, the morbidity rate was lower in the patients who underwent endoscopic biliary drainage than in those treated surgically, especially when complications such as chest infection, wound infection, and residual calculi were considered. We therefore think that emergency endoscopic biliary drainage should be offered to all patients with severe acute cholangitis due to choledocholithiasis.

Endoscopic biliary drainage may be accomplished in several ways. One is endoscopic papillotomy followed by active instrumentation for ductal clearance at the same session. ${ }^{1,3,4}$ Although such aggressive endoscopic therapy is probably the best way to secure adequate ductal drainage, patients with multiple large common-duct stones are not suitable candidates for such a procedure because it takes considerable time and has an increased risk of complications such as bleeding and retroduodenal perforation. ${ }^{1}$ Alternatively, temporary drainage can be established by the placement of a prosthesis. ${ }^{16}$ In our experience and that of others, ${ }^{2}$ endoscopic drainage with a nasobiliary catheter, which can usually be accomplished in 30 minutes, is a simple, safe, and effective measure for controlling biliary sepsis in properly selected patients.

In this study the mortality rate of 10 percent among patients with severe acute cholangitis treated with endoscopic biliary drainage was higher than that reported by others. ${ }^{1,2,4}$ Since endoscopic biliary drainage is a safe procedure, treating acute cholangitis early, without waiting for progression to severe disease, might be justifiable in selected patients. On the basis of our previous retrospective study ${ }^{7}$ and the results of this trial, the presence of concomitant medical problems, a low serum albumin concentration, and a low platelet count were risk factors for an unfavorable outcome, despite the use of therapeutic endoscopy. Although the presence and duration of septicemic shock correlated well with the development of thrombocytopenia, it was not possible to prevent or correct the remaining two factors. Even in the absence of fulminating biliary tract infection, urgent endoscopic intervention should be considered for patients who have any of these adverse prognostic determinants, once the diagnosis of acute calculous cholangitis is established.

\section{REFERENCES}

1. Leese T, Neoptolemos JP, Baker AR, Carr-Locke DL. Management of acute cholangitis and the impact of endoscopic sphincterotomy. Br J Surg 1986;73:988-92

2. Leung JWC, Chung SCS, Sung JJY, Banez VP, Li AKC. Urgent endoscopic drainage for acute suppurative cholangitis. Lancet 1989; 1:1307-9.

3. Delmotte IS, Pommelet P, Houcke P, et al. Initial duodenoscopic sphincterotomy in patients with acute cholangitis or pancreatitis complicating biliary stones. Gastroenterology 1982;82:1042. abstract.

4. Gogel HK, Runyon BA, Volpicelli NA, Palmer RC. Acute suppurative obstructive cholangitis due to stones: treatment by urgent endoscopic sphincterotomy. Gastrointest Endosc 1987;33:210-3.

5. Ikeda S, Tanaka M, Itoh H, Kishikawa H, Nakayama F. Emergency decompression of bile duct in acute obstructive suppurative cholangitis by duodenoscopic cannulation: a lifesaving procedure. World J Surg 1981;5:587-93.

6. Lai ECS, Paterson IA, Tam PC, Choi TK, Fan ST, Wong J. Severe acute cholangitis: the role of emergency nasobiliary drainage. Surgery $1990 ; 107$ : 268-72.

7. Lai ECS, Tam PC, Paterson IA, et al. Emergency surgery for severe acute cholangitis: the high-risk patients. Ann Surg 1990;211:55-9.

8. Blamey SL, Fearon KCH, Gilmour WH, Osborne DH, Carter DC. Prediction of risk in biliary surgery. Br J Surg 1983;70:535-8.

9. Boey JH, Way LW. Acute cholangitis. Ann Surg 1980:191:264-70.

10. O'Connor MJ, Schwartz ML, McQuarrie DG, Sumer HW. Acute bacterial cholangitis: an analysis of clinical manifestation. Arch Surg 1982;117:43741.

11. Welch JP, Donaldson GA. The urgency of diagnosis and surgical treatment of acute suppurative cholangitis. Am J Surg 1976;131:527-32.

12. Armitage P.The planning of statistical investigations. In: Armitage P, ed Statistical methods in medical research. Oxford, England: Blackwell Scientific, 1971:184-8.

13. Gigot JF, Leese T, Dereme T, Coutinho J, Castaing D, Bismuth H. Acute cholangitis: multivariate analysis of risk factors. Ann Surg 1989;209:435-8.

14. Pessa ME, Hawkins IF, Vogel SB. The treatment of acute cholangitis: percutaneous transhepatic biliary drainage before definitive therapy. Ann Surg 1987;205:389-92.

15. Kinoshita H, Hirohashi K, Igawa S, Nagata E, Sakai K. Cholangitis. World J Surg 1984;8:963-9.

16. Kiil J, Kruse A, Rokkjaer M. Large bile duct stones treated by endoscopic biliary drainage. Surgery 1989;105:51-6. 